\title{
Are postprandial responses influenced by melatonin production
}

\author{
V. Revell, M. Gibbs, K. Pudge, K. Stefanou, A. Hayashi and S. Hampton \\ Faculty of Health and Medical Sciences, University of Surrey, Guildford, Surrey GU2 7XH, UK
}

There is increasing evidence from animal studies that melatonin may have a role in glucose homoeostatasis ${ }^{(1)}$. Melatonin administration in normal weight rats has been shown to reduce body weight and intra abdominal adiposity ${ }^{(2)}$. However, the evidence for the role of melatonin on glucose in human subjects is scant although Peschke ${ }^{(3)}$ reported a reduction in melatonin secretion in diabetics and metabolic syndrome suggesting that the melatonin signal is essential for glucose homoeostasis and regulation. This study investigates the postprandial hormone and metabolic responses after a low glycaemic index (LGI) meal consumed in the late evening in the presence or absence of light. Light suppresses melatonin and therefore, in the bright-light condition melatonin levels would be low while in dim conditions ( $<5$ lux) melatonin levels would increase across the evening. It was hypothesised that in bright light conditions, when melatonin levels were low, postprandial insulin levels would be higher than under dark conditions, when melatonin is not suppressed by light.

In a randomised control, single-blind crossover study eight healthy subjects $(3 \mathrm{~F}, 5 \mathrm{M})$ mean age 21.5 year $(1.6 \mathrm{sD})$ with a $\mathrm{BMI} 21.4 \mathrm{~kg} / \mathrm{m}^{2} \mathrm{were}$ given an LGI meal at $18.00 \mathrm{~h}$ followed by further LGI meal at $23.00 \mathrm{~h}$ on two separate occasions. On the day of the study subjects had a control breakfast $(2585.71 \mathrm{~kJ}(1 \mathrm{kcal}=4.184 \mathrm{~kJ}))$ at $07.00 \mathrm{~h}$ and lunch $(3368.12 \mathrm{~kJ})$ at $13.00 \mathrm{~h}$. They arrived in the clinical unit at $18.00 \mathrm{~h}$ and were given a LGI meal $(2585.71 \mathrm{~kJ})$ followed by an LGI evening meal $(2397.43 \mathrm{~kJ})$ at $23.00 \mathrm{~h}$. Blood samples where obtained using the finger prick technique at regular intervals for $2 \mathrm{~h}$ starting directly prior to the consuming the evening meal. Actiwatches were worn by all subjects in order to monitor their activity and sleep-wake cycles. Subjects were asked to complete a $48 \mathrm{~h}$ urine collection the week prior to the study to assess the timing and levels of their melatonin rhythm using six sulphatoxymelatonin (aMT6), the major urinary metabolite of melatonin. Urine samples were also collected during the study to measure aMT6s levels. Plasma samples were analysed for glucose using the YSI 2300 analyser. TAG and NEFA were analysed using I-LAB 650 using enzymatic kits. Plasma insulin was measured by human specific RIA kit. aMT6 was measured using a competitive RIA. ${ }^{(4)}$ Comparison between total aMT6 production on each occasion was analysed by paired $t$-test. The postprandial hormone and metabolic responses were analysed by repeated measure ANOVA. $P<0.05$ was regarded as significant.

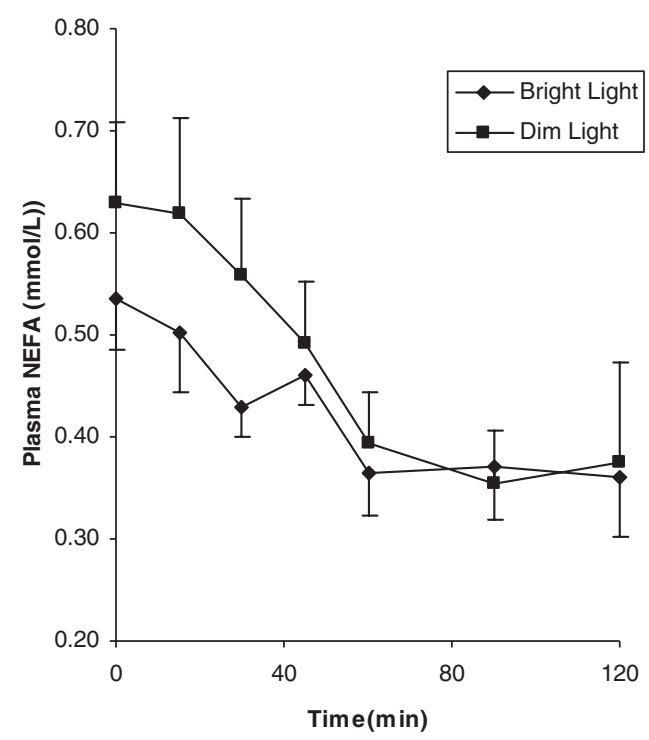

aMT6 output in five subjects (three had to be excluded due to incomplete data) showed a non-significant trend towards a reduction in melatonin output in the presents of bright light (2020 (4528) ng/h) compared with dim light (5591(1423) ng/h $P=0.07 n 5$ Mean(SE)). Postprandial glucose and TAG responses were not significantly different after the two treatments. Postprandial insulin area under the curves were not significantly different in dim light (2184(690) pmol/l) compared with bright light conditions (4937(2912) pmol/l $n$ 5). However, postprandial NEFA responses (Fig. $n$ 8) showed a trend towards a significantly lower response in bright light condition $(P=0.07)$.

The study has produced some interesting results regarding the depressed NEFA levels and a possible role of melatonin which requires further investigation. The study may have implications for those people working at night.

1. Sartori C, Dessen P, Mathieu C et al. (2009) Endocrinology 150, 5311-5317.

2. Rasmussen DD, Boldt BM, Wilkinson et al. (1999) Endocrinology 140, 1009-1012.

3. Peschke E, Stump I, Bazwinsky et al. (2007) J Pineal Res 42, 350-358.

4. Aldhous ME \& Arendt J (1998) Ann Clin Biochem 25, (Pt 3), 298-303. 